\title{
Analysis on the Current Situation and Future Development of Short Video Software ---Take Douyin as an Example
}

\begin{abstract}
Zihan $\mathrm{Su}^{* 1}$
${ }^{1}$ Shandong Agricultural University

*Corresponding author. Email: sufeng12345@163.com

ABSTRACT

In recent years, short video software has exploded, and various short video software such as Douyin has gradually become the most popular entertainment software. This article takes Douyin as an example to analyze the current situation and predict the future development of short video software. The article uses the Marketing Theory of 4Cs to analyze the customer's position, customer usage costs, platform operating costs, software convenience, and communication of the software. The existing problems of the short video software are found through analysis and some improvement measures are proposed. Finally, this paper collected and analyzed the data in recent years and concludes that the development of short video software will get better and better, and the penetration rate will also be higher and higher.
\end{abstract}

Keywords: Short video software, Douyin, The Marketing Theory of 4Cs, Data

\section{INTRODUCTION}

The development of short video software has an inseparable relationship with the development of the social environment. With the popularization of the Internet and smartphones, the number of china's mobile phone netizens has reached $93.6 \%$. This brings mobile phone software great development prospects. With the rapid development of society, we obviously feel the acceleration of the pace of life, the explosion of information, and the fragmentation of time. Under this change, more and more people do not have time to watch long videos, or are tired of that. Compared with reading text and pictures, people prefer to watch videos. Short videos were born and developed in this situation. This paper uses Douyin( Tik Tok ), the most popular online short video software in China, as an example for analysis. In the research process, the author uses $4 \mathrm{c}$ marketing theory to analyze the strategy and rationality of software marketing. The data used in this paper mostly comes from data websites, year-end reports and data officially released by Douyin. The purpose ofthis paper is to analyze the success reasons and the future of short video software such as Douyin, so as to provide certain experience for the industry and promote the development of the Internet industry.

\section{THE MARKETING THEORY OF 4CS----MARKETING STRATEGY ANALYSIS}

First of all, I will analyze the marketing strategy of Douyin based on the marketing theory of $4 \mathrm{Cs}$. The $4 \mathrm{C}$ marketing model is a consumer-oriented model proposed by Robert F. Lauterborn in 1993 based on the 4P model optimization. The purpose is to better adapt to the needs of mass marketing in blank marketing. The model sets four basic elements of the marketing mix: Customer, Cost, Convenience and Communication. And below I will use the 4C marketing theory to analyze Douyin.[1]

\subsection{Customer}

The core of $4 \mathrm{C}$ marketing theory is customers. Douyin can better meet customer needs and increase the stickiness of software users based on customer classification and intelligent distribution technology.

\subsubsection{Meet different types of customers' need}

Douyin meets multiple needs of users at the same time. Users can enjoy themselves by watching videos 
for leisure and entertainment, and at the same time, they can obtain a variety of information and knowledge by watching videos. There are not only funny videos on Douyin, but also various teaching videos. Whether it is dancing, cooking, English, or life skills, you can find whatever you want on Douyin. Users can upload videos to show themselves. Many ordinary people with unique skills upload videos to let more people see themselves and become famous. At the same time, users can also make money in various ways. For example, you can build your own brand, accept advertising, sell goods, and even help farmers sell unsalable agricultural products.

\subsubsection{Users can find friends they know by discovering friends}

Users can find friends they know or may know people through the friend discovery FfamiliaFFr APPs. This not only meets the sharing needs of users, but also expands the customers of the software, such as the potential users who haven't used Douyin before may download the software and use it because of the sharing with friends.

\subsubsection{Intelligent push----push videos that users are interested in through big data analysis}

The Douyin software intelligently pushes videos through big data analysis. The software first automatically categorizes videos. By using big data technology, it distinguishes real consumers from potential consumers, and tags fans by gender, age, and region to build users' portraits. On the basis of customer portraits, Douyin provides personalized hot videos that users are interested in. At the same time, users can push more similar or related videos through the analysis of the types and the time of videos that users have watched. This can more accurately grasp the preferences of customers, which can attract customers to watch.

\subsubsection{Users have a dual identity--spreader and recipient}

Douyin's users can be simple spreaders or simple recipients. It can also have the dual identities of the spreader and recipient.[2]

As a spreader, Douyin's video shooting and production are relatively easy, with many good-looking special effects that meet the user's aesthetics, so that more users are willing to shoot and share. Douyin's slogan is to "share the good life". Such a platform gives ordinary people an opportunity to show their self-sharing life. Shooting videos at the same time have the possibility of gaining fame and even making profit. When the video becomes popular and the number of viewers is large, video bloggers can have a variety of ways to make money from traffic. For example, opening an online shop, receiving advertisements and so on. This encourages video bloggers to upload more videos and shoot more videos with good quality and creative content to attract more fans and maintain their activeness.

As a recipient, short videos abandon the logic and form of traditional videos in the past, and can quickly attract users' attention, and convey a variety of different content to users through interesting and short videos. Since most of the makers of videos are ordinary people, it will be easier to shoot videos that can meet the public love and see from the perspective of the audience, as a result, it will easily achieve a good dissemination effect. The public can enrich their fragmented time by watching short videos that count in seconds. According to the data analysis of the big data system, the videos which are related to the videos customers may like will be recommended to the customers, so it can attract the long-term use of the recipient and make the user sticky.

\subsection{Cost}

Cost is not only the production cost of the company, or the Price in 4P, it also includes the customer's purchase cost, It also means that the ideal price of the product should be lower than the customer's psychological price, and it can also need to ensure that the company can make enough profit. In addition, the purchase cost of the customer not only includes monetary expenditure, but also the time, physical and energy consumed for this purpose, and the purchase risk.

\subsubsection{Costs paid by consumers}

\subsubsection{Time cost}

First of all, as a video software, customers choose it based on the increase of fragmented time, and there is not much time for some people to watch long videos and long articles, but the duration of short videos is often only a few seconds to a few minutes. These videos can be watched in a short time and the content or information in them can be received quickly. Therefore, choosing to watch short videos can reduce the user's time cost.

\subsubsection{Risk cost}

Neither of using the short video APP, watching short videos and shooting short videos cost any money, even if there are a series of places where you can spend money, such as short video rewards, window merchandise purchases, live broadcast rewards, and merchandise purchases, but all of them are completely due to the customers' volunteer. General users can still watch short videos and live broadcasts without spending 
a penny, which does not affect the use of most users, so the risk cost is also very low.

\subsubsection{Spiritual cost}

Short video software such as Douyin have a very good data analysis and customer classification system. It can more accurately discover the content that customers prefer, and push related content or similar content to the customers which make the customers do not need to find videos they prefer to watch by themselves. Even if they want to find specific aspects of content, they can also search with some keywords, and they can also choose to look through the hot search list in Douyin to find the big events happened these days. All these functions I talked about can save the spiritual cost of customers.

\subsubsection{Physical cost}

The feature of using the video APP is that there is almost no physical cost. Video viewers do not need any physical consumption besides swiping the video to watch the video. Douyin has very good special effects and beauty functions, so it is easy to shoot nice videos. From these two parts I talked about, we can find that the physical cost of Douyin APP consumers is also very low.

\subsubsection{Platform: Zero cost of traffic monetization and launch}

We first introduce a concept of KOL (key opinion leader), KOL is a marketing concept which is usually defined as the people who have more accurate product information, and they are accepted or trusted by some related groups. And they have a big influence to the purchase behaviors of these groups. In the short video era, KOL usually are the stars, live streamers, Internet celebrities, self-media bloggers and so on. The products sold by the KOL through short videos software like Douyin are required to pay $6 \%$ of the fee to the Douyin platform as a commission. The platform does not need to pay for traffic monetization or product launches, but they can still receive a corresponding commissions.

\subsection{Convenient}

\subsubsection{Easy to use and operate:}

The low threshold--everyone can upload a video and everyone can be a star.

Compared with long videos, short videos have a lower threshold for creation, the software is very easy to use so you can easily shoot videos and upload them. The software simplifies the video production process and technology so that everyone can easily take video shots, thus attracting more users.

\subsubsection{Easy to be received}

In modern society, because of the developed Internet, more and more information comes out. Therefore, people need to obtain information more and faster. Compared with text and pictures, videos have the characteristics which can show more and quicker information. And short videos are only a few seconds or a few minutes which is more attractive to users than long videos. So short videos perfectly meet the needs of people in modern society for quick access to information.

\subsection{Communication}

In traditional video software, it is difficult for customers to directly communicate and interact with celebrities and KOLs. However, the high-frequency and strong interaction mode adopted by Douyin allows customers to directly communicate with the video content publisher or other users who watched the videos. And the users can also send private messages to another users include celebrities. Moreover, users can also get the public's views and opinions on the video content through the comments under the videos. For example, some beauty bloggers will recommend some products, and we can find some comments written by people who have used this product which is really helpful for customers to make better judgments on the products. This interactive mode can make the communication of customers more convenient and meet the needs of modern society.

\section{THE BUSINESS MODEL OF SOFT VIDEO SOFTWARE}

\subsection{Find the Develop Direction of the App Accurately}

\subsubsection{Position the content}

In the beginning, the videos of Douyin were mostly creative videos, people who uploaded the video paid more attention to the innovation of video content. These videos are very interesting and fashionable, so they are liked by many young people. With the development of Douyin products, the video content becomes more and more refined. There are more kinds of information in the videos, such as dance, make-up, cooking, finance, parenting, fitness, medical treatment, education, beauty and so on. Almost everything you can think of can be found on Douyin. Therefore, the content of Douyin products has a good quality and quantity, which can meet the needs and desires of various customers. 


\subsubsection{Position the users}

The initial age of Douyin's user group is positioned as young people, and the product is positioned as a short music video community for young people. The goal audience of Douyin at first is positioned as people who lived in the first-tier cities. Because young people in first-tier cities have a faster pace of life, they prefer to spend more fragmented time to receive information or take rest. And they also inclined to pursue fashion and trends. The gameplay, special effects and music designed by the Douyin software are sufficient to meet the needs of these young people. Therefore, it attracted a large number of users in the early stage of its launch.
Many people born in the 90s and 00s began to use Douyin. After March 2018, Douyin replaced slogan with "Recording a Good Life", which indicates that Douyin has adjusted its positioning of the user group and is no longer limited to young people in first-tier cities. It is already stabilizing and consolidating products. The audience expands to people of all ages and cities. And the picture below is the current distribution of Douyin's users. We can see the strategy runs very well, so now Douyin have all ages, cities users.

\section{Douyin User Portrait}

\section{Gender distribution age distribution city distribution}
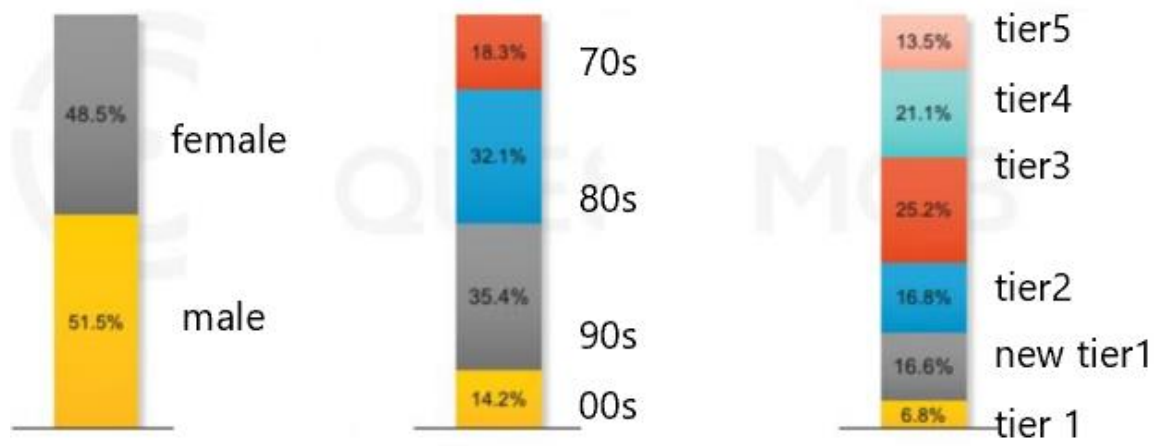

Figure1 The portrait of Douyin's users nowadays[7]

\subsection{The Overall Operation of the Product at the Initial Stage}

In the early stage of operation, Douyin recruited about 300 Internet celebrity seed players to create unified training content, attracting Internet celebrities who have a strong desire to display themselves and desire a platform, they produced excellent works to attract people to use this software. At the same time, celebrities and stars are invited to settle in the platform, which can attract their fans to use Douyin. Douyin is different from Kuaishou (another popular Chinese short video software) in terms of operating strategy. Kuaishou adopts a decentralized marketing strategy which means that the works in Kuaishou mainly are general people. Such a product positioning allows more ordinary people to shoot the videos and upload online. And everyone who uploads the video has the same opportunity to show themselves which means the platform will not bias to some specific users. But Douyin adopts a top-down operation method driven by celebrities, which means inviting celebrities to join and drive their fans to consume. But at the same time, it also takes into account the decentralization strategy to drive ordinary people to actively create videos or watch videos. While Kuaishou only uses the decentralization strategy. As a result, Douyin is developing faster than Kuaishou.

\subsection{The Success of Establishing a Topic Model}

The community development of Douyin is very good. He is the first to develop community-based short video software. His topic function is also one of his excellent business strategies. Everyone can initiate a topic, and everyone can participate in it. When a topic becomes famous, many people will follow this topic and take the same or related videos of it. Topics can guide users well. When a topic is popular, thousands of followers will take pictures, which will result in more excellent works and more user remakes. It not only can guide users well, but it also can be used for publicity. Some stars will initiate topics related to their new works, such as dance challenges, so that they can promote their works well. 


\section{THE EXTENSION DEVELOPMENT OF SHORT VIDEO APP}

\subsection{Live Stream-----the Live streamer Interaction/Live-stream Shopping}

Douyin not only has a variety of short videos, but also developed a live broadcast function. Everyone can use their own account to live broadcast. The live streamers are divided into various types, including entertainment live streamers, game live streamers, outdoor live streamers and shopping live streamers. Entertainment live streamers are the most primitive live streamers. Most live streamers have certain talents, good looks, and attractive personalities. There are also different types of game live streamers. Some play games live, and some report game events. Many game enthusiasts like to watch this kind of live broadcast. Outdoor live streamers refer to the live streamers that conduct live broadcasts outdoors, with various forms of expression, singing, dancing, and displaying natural scenery. The most distinctive live broadcasts are outdoor adventures and outdoor survival live broadcasts. The first three kinds of live streamers mostly get money through platform rewards by the Platform users. And the last kind of live streamers is getting more and more popular. They sell various products through live broadcasts, and most of the products have good discounts. The live streamers use the products on live to attract customers to buy them. Now Douyin has become a very important live streaming platform in China.

To be specific, I will introduce Yonghao Luo, the biggest personal shopping live streamer in Douyin as an example. Douyin's live broadcast sales during Double Eleven (10/25-11/11) in 2020 were RMB 18.7 billion, and the team of Luo Yonghao personal sales during Double Eleven had reached RMB 300 million.

\begin{tabular}{l|l|l|r|r|} 
live streamer & $\begin{array}{l}\text { Number of } \\
\text { followers }\end{array}$ & $\begin{array}{l}\text { Number of } \\
\text { live } \\
\text { broadcasts }\end{array}$ & $\begin{array}{l}\text { Average } \\
\text { sales per } \\
\text { game(RMB) }\end{array}$ & $\begin{array}{l}\text { Cumulative } \\
\text { sales(RMB/ } \\
\text { billion) }\end{array}$ \\
\hline SUNING & $2,763,000$ & 14 & $38,382,000$ & 0.54 \\
\hline Yonghao Luo & $13,186,000$ & 10 & $34,337,000$ & 0.34 \\
\hline Beibei Rabit & $10,042,000$ & 16 & $12,253,000$ & 0.2 \\
\hline Yige & $16,946,000$ & 11 & $17,606,000$ & 0.19 \\
\hline Haiquan Hu & $2,493,000$ & 3 & $58,680,000$ & 0.18 \\
\hline
\end{tabular}

This is the sales of various commodities during Double Eleven(10.25-11.11). It can be seen

that the sales of clothing, shoes and bags, beauty care products, and catering food are the and child products, home furnishings, cultural and entertainment products and outdoor

sports products.

top ones, followed by digital appliances, watches and jewelry, daily necessities, Maternal

\begin{tabular}{|c|c|c|c|c|c|}
\hline Category Information & $\begin{array}{l}\text { Number of } \\
\text { Products }\end{array}$ & $\begin{array}{l}\text { Number of } \\
\text { live } \\
\text { streamers }\end{array}$ & $\begin{array}{l}\text { Number of } \\
\text { live } \\
\text { broadcasts }\end{array}$ & $\begin{array}{l}\text { Cumulative } \\
\text { sales }\end{array}$ & $\begin{array}{l}\text { Cumulative } \\
\text { sales (RMB/ } \\
\text { billion) }\end{array}$ \\
\hline Clothing, shoes and bags & $2,470,000$ & 14,000 & 82,000 & $25,644,000$ & 2. 95 \\
\hline Beauty products & 43,000 & 14,000 & 57,000 & $13,220,000$ & 1. 16 \\
\hline Catering food & 36,000 & 22,000 & 102,000 & $26,771,000$ & 1. 07 \\
\hline Digital appliances & 9381 & 11,000 & 43,000 & $1,761,000$ & 0.68 \\
\hline Watches \& Jewellery & 31,000 & 3999 & 15,000 & $4,006,000$ & 0.41 \\
\hline Daily necessities & 34,000 & 15,000 & 65,000 & $9,109,000$ & 0.35 \\
\hline Baby Products & 33,000 & 6390 & 28,000 & $5,269,000$ & 0.24 \\
\hline Furniture home improvement & 17,000 & 4796 & 22,000 & $1,894,000$ & 0.16 \\
\hline Cultural and entertainment products & 10,000 & 8477 & 38,000 & $2,330,000$ & 0.11 \\
\hline Sports outdoor products & 6983 & 2967 & 15,000 & 851,000 & 0.060062 \\
\hline
\end{tabular}

Figure3 2020 (10.25-11.11) sales of various commodities[4]

\subsection{The Shop Window of the E-commerce}

As Douyin becomes more and more well-known and has more and more users, the Douyin platform has launched a product window[3], which is displayed below the short video, and the video uploader can upload the shop window and video at the same time. So this function adds an opportunity to make money for users who upload videos. Many merchants and Internet celebrities also use this method to sell goods. For example, a clothes seller can wear their clothes and shoot a good-looking video, and there will be many viewers who like these clothes to buy them in the window. The sales volume of this kind of e-commerce window is very impressive, which makes the platform and merchants profit a lot and it also promotes the development of the interactive economy. 


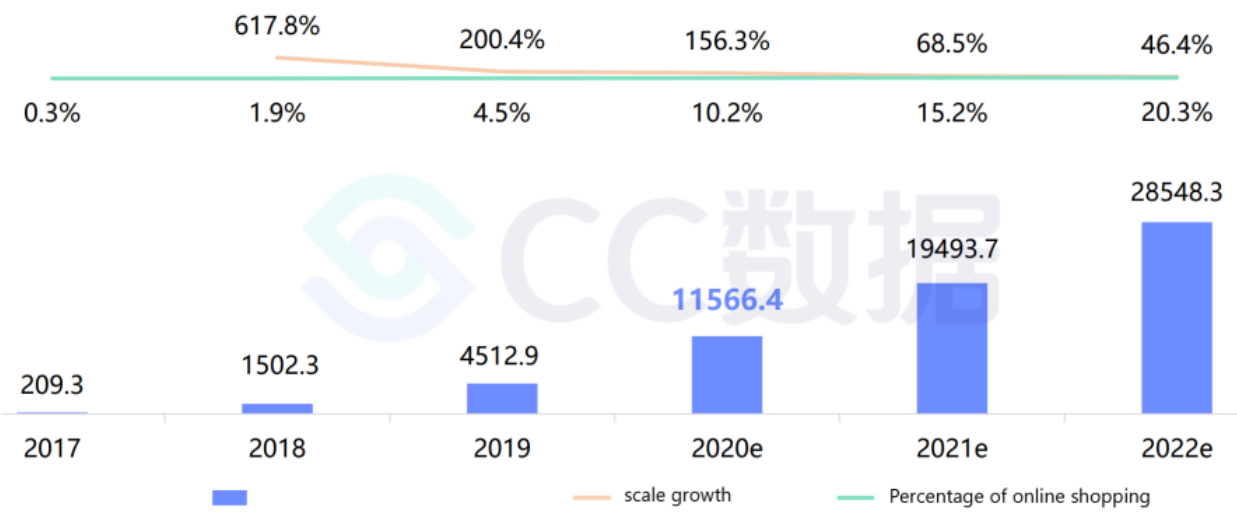

2017-2020 China's live broadcast e-commerce transaction scale and growth rate

source: iResearch

Figure4 2017-2020 China's live broadcast e-commerce transaction scale and growth rate[4]

\section{DOUYIN'S CURRENT PROBLEMS AND SOLUTIONS}

\subsection{The Mechanism of Merchant Review is not Perfect: It Hinders Sustainable Development}

Due to the low cost of short video operation and low operating threshold, a large number of businesses have settled in Douyin. However, the current Douyin review mechanism is not perfect.[5] There is a certain gap between the products shown in the videos uploaded by some merchants and the actual products, resulting in a product price and quality mismatch, and the software refund mechanism is not perfect. These problems will make users lose the trust of some merchants even to the APP, which will hinder the sustainable development of Douyin software. If we want to make Douyin's product sales function have a sustainable development, developers should set up a strict review mechanism to better review both the merchants and products, and create a sales model with quality, quality and reputation.

\subsection{The Homogeneity of Video Content is Serious: It is Easy to Produce Aesthetic Fatigue}

The content of Douyin short videos is now very homogenous. There are often creators who copy other creators' videos in pursuit of more video views.[6] There are also many bloggers with a large number of fans who publish videos that are very similar. Original videos are relatively too little, which will cause viewers to suffer aesthetic fatigue for such type of short videos. Douyin should encourage more creators to publish original works, reward popular original works, and penalize creators who plagiarize videos without indicating the original source. This can increase the diversity of video content and attract more users. His will also effectively increase the user stickiness and the number of time users spend using the app.

\section{THE DEVELOPMENT SITUATION AND THE PREDICT OF THE SHORT VIDEO APP}

The two figures below show us the DAU and MAU of different kinds of APP which is popular today.

DAU: daily active users

MAU: monthly active users

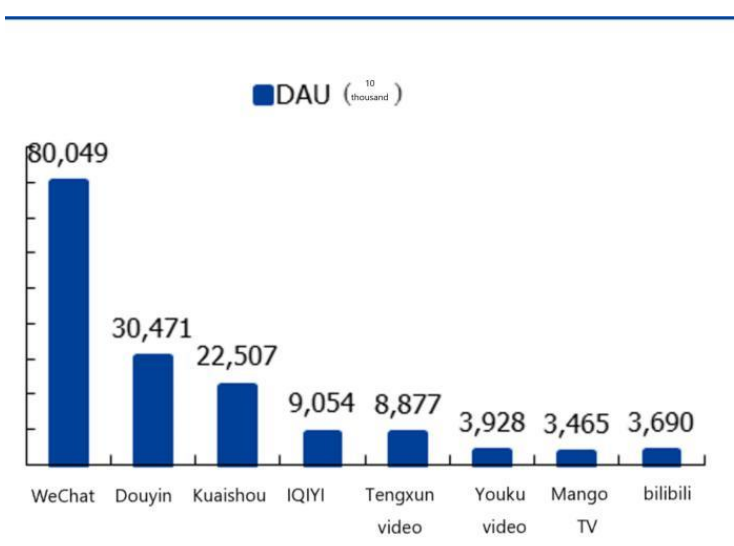

Figure5 DAU of different APP[7] 


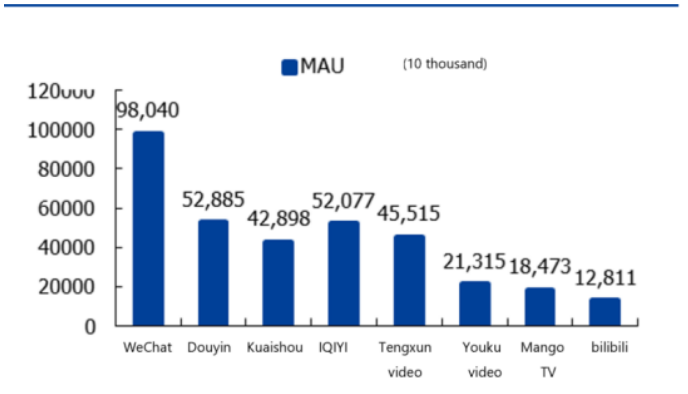

Figure6 MAU of different APP[7]

By analyzing these two charts, we found that Douyin has a very large number of active users, second only to WeChat, our most frequently used chat app. Therefore, the short video software Douyin is very popular among users used by many people for leisure and entertainment.

2019.08 and 2020.08 average monthly usage time per user in the entertainment industry

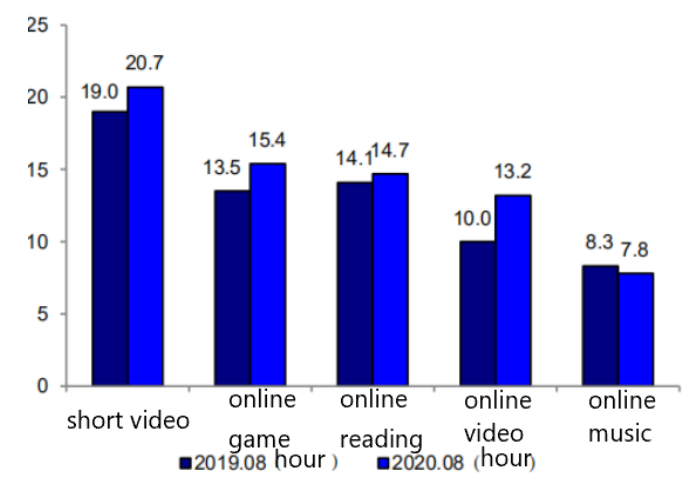

Figure7 Average monthly usage time per user in the entertainment APP
We divide leisure and entertainment software into short video software, online game

software, online reading software, online video software and online music software. Through

this chart, we find that short video software has become the most popular leisure and

entertainment software. People spend more time watching short videos, so I include that

watching short video is a very common way of entertainment in modern society.

\begin{tabular}{|c|c|c|c|c|c|}
\hline & \multicolumn{2}{|c|}{2020.3} & \multicolumn{2}{|c|}{2020.12} & $\vdots$ \\
\hline users & $\begin{array}{l}\text { *10000 } \\
\text { User scale }\end{array}$ & $\begin{array}{l}\text { Utilization rat } \\
\text { of netizens }\end{array}$ & $\begin{array}{l}e^{*} 10000 \\
\text { user scale }\end{array}$ & $\begin{array}{l}\text { Utilization rate } \\
\text { of netizens }\end{array}$ & growth rate \\
\hline $\begin{array}{l}\text { Network video } \\
\text { (including short video) }\end{array}$ & 85044 & $94.1 \%$ & 92677 & $93.7 \%$ & $9.0 \%$ \\
\hline short video & 77325 & $85.6 \%$ & 87335 & $88.3 \%$ & $12.9 \%$ \\
\hline
\end{tabular}

Figure8 The user scale and the utilization rate of netizens.

This chart shows the scale of short video users and their proportion to netizens in March 2020 and December 2020. We found that the number of users of short video software is increasing and it accounts for a large proportion of netizens.

\section{CONCLUSION}

Through the above data analysis, this paper found that the active users of Douyin have reached a large scale and the number of users is increasing. According to the data analyzed in the user positioning section, it can be found that the user range of Douyin is also constantly increasing. Of course, there are still some problems in the software, but they can all be compensated with the continuous improvement of developers. Therefore, in the future, short video software such as Douyin will have a greater market and better development prospects.

\section{ACKNOWLEDGMENT}

First and foremost,I would like to show my deepest gratitude to my teachers and professors in my university, who have provided me with valuable guidance in every stage of the writing of this thesis.Further,I would like to thank all my parents, friends and roommates for their encouragement and support.Without all their enlightening instruction and 
impressive kindness, I could not have completed my thesis.

\section{REFERENCES}

[1] Zhong Ziyin. "Short video +" marketing model and strategy research [D]. Jiangxi Normal University, 2020.

[2] Cheng Shiruo, Lou Zilong. Research on the dissemination characteristics and dissemination effects of "Douyin" APP [J]. Shanxi Youth, 2020(11): 68-69+119.

[3] Wang Guiwen, Pan Qixin, Tang Xingji. Research on the planning and marketing strategy of short videos_-Taking the Douyin platform as an example [J]. News Frontier, 2021(01): 39-40.

[4] Chen Xuefang. Analysis of the reasons for the popularity of short video APP and its development_-Taking "Tik Tok" as an example[J]. Audiovisual, 2018(11): 136-137.

[5] Zhao Xinqi. Analysis of the profit model of Douyin APP [J]. Business Economics, 2020(10): 72-73.

[6] CCsight. Douyin marketing data report during Double Eleven in 2020.https://ccsight.cn

[7] Data source: Questmobile 